\title{
Extra nodal Rosai Dorfman disease of nasal septal mucosa without lymphadenopathy
}

\author{
Raje $\mathrm{P}^{1}$, Vyas $\mathrm{P}^{1}$ \\ ${ }^{\text {I} D e p a r t m e n t ~ o f ~ S u r g i c a l ~ P a t h o l o g y ~ a n d ~ C y t o l o g y, ~ B o m b a y ~ H o s p i t a l ~ I n d o r e ~-~ I n d o r e, ~ I n d i a . ~}$
}

\section{Keywords: \\ Nasal septum; \\ Rosai-Dorman disease; \\ Lymphadenopathy}

\begin{abstract}
Rosai-Dorman disease, also known as sinus histiocytosis with massive lymphadenopathy, typically presents as massive enlargement of lymph nodes accompanied by systemic symptoms. Extranodal involvement is also known usually in head and neck regions. Rarely the disease presents at extranodal sites only without involvement of lymph nodes.

We present a case of 58 years-old female who presented with mass in nasal septum without involvement of lymph nodes. The mass was removed surgically and diagnosis was made on histological grounds with classical findings of histiocytic proliferation and emperipolesis.
\end{abstract}

\section{INTRODUCTION}

Rosai-Dorman disease (RDD), also known as sinus histiocytosis with massive lymphadenopathy (SHML), a rare and usually self limiting disease, was originally described by Destombes in $1965 .{ }^{1}$ Subsequently; in 1969, it was characterized as a distinct clinicopathological disorder by Rosai and Dorfman. ${ }^{2}$ It is a pseudolymphomatous nonneoplastic lymphoproliferative disease of unknown etiology that presents with systemic symptoms. ${ }^{3}$ The presentation is bilateral cervical, axillary or inguinal lymphadenopathy that is painless, accompanied with pyrexia, leukocytosis and hypergammaglobulinemia. ${ }^{3}$

\section{Correspondence:}

DR. Prakash Raje. MD DCP

Senior Consultant and Head, Department of Surgical Pathology and Cytology, Dr Prakash Raje, 271, Saket, Indore, MP, India, 452018.

Email:drprakashraje@gmail.com
In $43 \%$ cases disease may have extra nodal involvement ${ }^{4}$, most common sites being nasal cavities, skin, eyes, orbit, eyelids, bones, soft tissue, the central nervous system, upper respiratory tract and less commonly, the gastrointestinal tract, But it may present itself at extra nodal sites only in absence of obvious lymphadenopathy. ${ }^{5-11}$

Rosai-Dorman disease typically manifests in childhood or in early adulthood, mostly in the second and third decades of life. ${ }^{12}$ Caucasians have a higher prevalence than African Americans. A male predominance is observed. ${ }^{12}$ RosaiDorman disease is rare in India, till 2007 only about a dozen cases have been reported, all in children and none in adults. No case of extra nodal involvement has been reported. The disease usually follows an insidious course but could be fatal due to involvement of vital organs. 


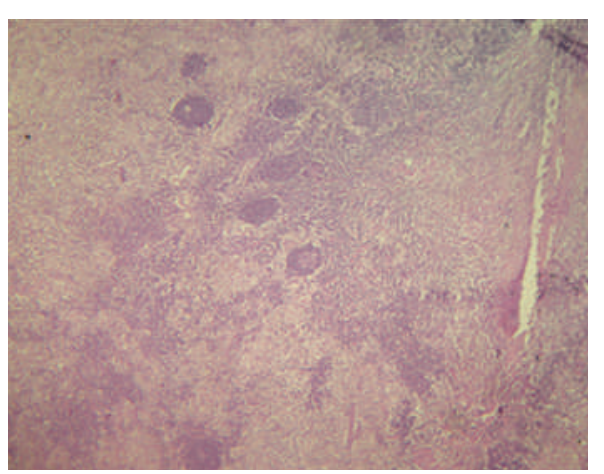

Figure 1: Low power view of the lesion, nasal mucosa with histiocytic proliferation underneath; Extensive histiocytic proliferation. And few lymphoid follicles [HE stain, X100]

Histopathologically, it is characterized by flooding of lymphatic sinuses by histiocytes and plasma cells. The most important diagnostic finding is emperipolesis which is active penetration of a smaller cell into or through a larger cell more commonly phagocytosis of intact lymphocytes, plasma cells or red blood cells by histiocytes. ${ }^{13}$ In extranodal diseases emperipolesis is seen less frequently but in our case it was quite frequent. Immunohistochemistry like positivity for $\mathrm{S} 100$ and $\mathrm{CD} 68(+)$ and negativity with $\mathrm{CD} 1$ a protein help differentiate RDD from malignant disorders such as lymphoma.

\section{CASE REPORT}

A 58-year-old female patient presented to the outpatient department with complaints of difficulty in breathing and frequent episodes of epistaxis since three months. Endoscopic nasal examination revealed a mass originating from the nasal septal mucosa.

Computed tomography images of right paranasal sinus showed a large well defined homogenously enhancing soft tissue density mass lesion in the right half of nasal cavity measuring $5 \times 3.7 \times 2.5 \mathrm{~cm}$ polyp arising from right nasal septum. The lesion was causing expansions of the nasal cavity with smooth scalloping and remodeling of adjacent bones. It was obliterating all drainage pathways. Left nasal cavity and paranasal sinus were unremarkable.

The mass was endoscopically excised which was soft in consistency, largest $3.5 \times 3 \times 1$ centimeters. The histopathological examination revealed a polypoidal lesion lined by psuedostratified columnar epithelium. Stroma showed sheets of histiocytes along with a large number of mature plasma cells, lymphocytes and few lymphoid follicles with prominent germinal centre.(fig.1) Most of the histiocytes did not show any foamy cytoplasm. Bi and multinucleation was also seen, however popcorn cells were absent. There was no fibrocollagenous proliferation. Many

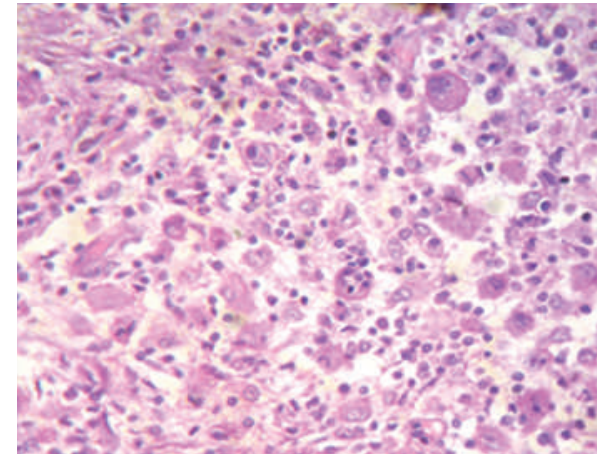

Figure 2: High power view of the lesion, extensive emperipolesis. [HE srtain, X400]

histiocytes had large and vesicular nuclei and showed lymphocytic emperipolesis. (fig.2). The histiocytes were S100(+), CD68(+) and negatively stained with CD1a. Histopathological and immunohistochemical findings were consistent with the diagnosis of RDD- extranodal involvement.

\section{DISCUSSION}

Rosai-Dorman disease is a disease of unknown etiology. Histiocytic proliferation could be due to a long standing occult infection or an altered immune response against an antigen. Epstein Barr Virus and Human Herpes Virus- $6^{11}$ have also been implicated in the pathogenesis of SHML. It could be a disorder of the mononuclear and immunoregulatory effect system with a speculated association with EBV and HHV-6. ${ }^{11,12-14}$ Expression of HHV-6 antigen has been identified in the histiocytes present in RDD, while lymphocytes express EBV and parvovirus. Eventually the infected lymphocytes are phagocytosed by histiocytes. ${ }^{14-15}$

Differential diagnosis of RDD involves other causes of lymphadenopathy especially nonmalignant conditions like tuberculosis, reactive sinus hyperplasia. Wegener's granulomatosis, sarcoidosis, IgG4-related disease, juvenile xanthogranuloma, Gaucher's disease and other disorders of histiocytic proliferation such as Langerhans cell histiocytosis.

Malignant conditions causing lymphadenopathy include Hodgkin's lymphoma, non-Hodgkin lymphoma, melanoma, leukemia, and Langerhans cell sarcoma. In addition, due to the localization and the development of a nasal mass, primary nasopharyngeal carcinoma and olfactory neuroblastoma should also be considered in the differential diagnosis.

Clinical, laboratory and histopathological findings 
considered together help differentiate chronic inflammatory diseases such as tuberculosis, sarcoidosis and reactive sinus histiocytosis. A marked difference in the histopathology and simultaneous CD4 and CD8 positivity exclude Hodgkin's and non Hodgkin's lymphoma. Langerhans cell histiocytosis is differentiated from RDD by the absence of emperipolesis and CD1a positivity. Nasopharyngeal carcinoma is differentiated by means of cell type and its aggressive behavior.

RDD is usually a self limiting benign disease. Most of the cases do not require any treatment ${ }^{16} \mathrm{In}$ few cases it follows a more aggressive course due to widespread involvement of vital organs. In occasional cases a fatal outcome is also reported. Treatment options in non self limiting cases or those with massive enlargements of lymph nodes or extranodal sites are steroids, chemotherapy, radiation, monoclonal antibody treatment, acyclovir, interferon and thalidomide.

Surgery is usually undertaken when the mass causes a functional impairment in an organ or is life threatening. This case presented with nasal obstruction and difficulty in breathing and was subjected to endoscopic removal of the mass. No other treatment was given.

\section{CONCLUSION}

Since most of the times RDD is a benign self limiting disease that does not require any aggressive treatment it is imperative to differentiate it from other causes of lympadenopathy. For the same reason both clinicians and pathologists should have a high degree of suspicion for patients presenting with generalized lymphadenopathy with/without extra nodal involvement or masses with histiocytic proliferation in extranodal sites. Presence of emperipolesis is an important diagnostic finding.

\section{REFERENCES}

1. Destombes P. Adenitis with lipid excess, in children or young adults, seen in the Antilles and in Mali. (4 cases) [in French]. Bull Soc Pathol Exot Filiales. 1965;58:1169-75.

2. Rosai J, Dorfman RF. Sinus histiocytosis with massive lymphadenopathy. A newly recognized benign clinicopathological entity. Arch Pathol.1969;87:63-70. Crossref

3. J. Rosai and R. F. Dorfman, "Sinus histiocytosis with massive lymphadenopathy. A newly recognized benign clinicopathological entity," Archives of pathology, 1969;87:63-70, Crossref

4. Foucar E, Rosai J, Dorfman R. Sinus histiocytosis with massive lymph-adenopathy (Rosai-Dorfman disease): review of the entity. Semin Diagn Pathol. 1990;7:19-73. Crossref
5. Cooper SL, Jenrette JM. Rosai-Dorfman disease: management of CNS and systemic involvement. Clin Adv Hematol Oncol. 2012;10:199-202. Crossref

6. Frater JL, Maddox JS, Obadiah JM, et al. Cutaneous Rosai-Dorfman disease: comprehensive review of cases reported in the medical literature since 1990 and presentation of an illustrative case. J Cutan Med Surg.2006;10:281-90. Crossref

7. Kong YY, Kong JC, Shi DR, et al. Cutaneous Rosai-Dorfman disease: a clinical and histopathologic study of 25 cases in China. Am J Surg Pathol.2007;31:341-50. Crossref

8. McClellan SF, Ainbinder DJ. Orbital Rosai-Dorfman disease: a literature review. Orbit 2013;32:341-6. Crossref

9. Purav P, Ganapathy K, Mallikarjuna VS, et al. Rosai-Dorfman disease of the central nervous system. J Clin Neurosci. 2005;12(6):656-659. Crossref

10. Sandoval-Sus JD, Sandoval-Leon AC, Chapman JR, et al. RosaiDorf- man disease of the central nervous system: report of 6 cases and review of the literature. Medicine (Baltimore). 2014;93:165-75. Crossref

11. Zhao M, Li C, Zheng J, et al. Extranodal Rosai Dorfman disease involving appendix and mesenteric nodes with a protracted course: report of a rare case lacking relationship to IgG4-related disease. Int J Clin Exp Pathol. 2013; 6: 2569-77. Crossref

12. G. Pettinato, J. C. Manivel, E. S. G. d'Amore, and G. Petrella, "Fine needle aspiration cytology and immunocytochemical characterization of the histiocytes in sinus histiocytosis with massive lymphadenopathy (Rosai-Dorfman syndrome)," Acta Cytologica 1990;34:771-7. Crossref

13. Mehraein Y, Wagner M, Remberger K, et al. Parvovirus B19 detected in Rosai-Dorfman disease in nodal and extranodal manifestations. J Clin Pathol.2006;59:1320-6. Crossref

14. Luppi M, Barozzi P, Garber R, et al. Expression of human herpesvirus-6 antigens in benign and malignant lymphoproliferative diseases. Am J Pathol.1998;153:815-23. Crossref 